\title{
Local smoothing properties of a Schrödinger equation with nonconstant principal part
}

\author{
Mary Ann Horn* \\ Department of Mathematics, Vanderbilt University \\ Nashville, Tennessee 37240, U.S.A. \\ and \\ Walter Littman \\ School of Mathematics, University of Minnesota \\ Minneapolis, Minnesota 55455, U.S.A.
}

\begin{abstract}
A Schrödinger equation with continuous, nonconstant coefficients appearing in the principal part of the differential operator is considered. Through the use of Littman and Taylor's general technique for proving boundary controllability of evolution equations, a simple proof of exact boundary controllability for the Schrödinger equation is obtained. Proof of the necessary regularity for the solution relies on an approximation argument and the spectral results for elliptic operators of Birman and Solomyak.
\end{abstract}

Keywords

Schrödinger equation, exact controllability, regularity.

\section{INTRODUCTION}

In the context of controllability, local smoothing properties for the Schrödinger equation were first addressed in Littman and Taylor (1992) where the Schrödinger equation with nonsmooth potential was considered. In this work, a very general technique for proving boundary controllability was developed which is applicable to many classes of equations. It can be summed up in the following way:

$$
\text { local smoothing }+ \text { uniqueness }+ \text { reversibility } \Longrightarrow \text { exact boundary controllability. }
$$

Here "uniqueness" is the uniqueness property implying approximate controllability by duality. If control is to be exercised on the whole lateral boundary of a cylindrical domain,

\footnotetext{
*This material is based upon work partially supported under a National Science Foundation Mathematical Sciences Postdoctoral Research Fellowship.
} 
$\Omega \times(0, T)$, in space-time, this means that every solution (in an appropriate space) of the homogeneous linear evolution equation in this domain having zero Chauchy data on the lateral boundary must vanish identically. "Reversibility" means that the backward problem (in time) is wellposed. For the Schrödinger equation, the difficulty of applying Littman and Taylor's approach lies in proving the necessary smoothing properties.

Although various systems of equations have been considered and a variety of techniques developed in the context of boundary control, the appeal of this method is its generality and avoidance of many of the technicalities of other related works. In comparison, Lasiecka and Triggiani (1992) use the method of multipliers to obtain boundary controllability for the Schrödinger equation with constant coefficients. However, the estimates involved are very sensitive to the lower order differential terms which will be inevitably produced by the variations of the coefficients. While some oscillations of the coefficients can still be handled by the existing techniques, to treat the general case, new approaches need to be developed.

In Lebeau (1992), the results of his work with Bardos and Rauch (1989) on exact controllability for hyperbolic equations are modified and applied to Schrödinger type equations. However, in the statement of his results, Lebeau assumes constant coefficients and an analytic boundary. Although his work appears to extend to the case of nonconstant coefficients, our goal, as already stated, is to avoid the technical difficulties of applying his technique to the more general case.

We note that this paper is a preliminary report. A more detailed and comprehensive treatment, including regions other than $\mathbf{R}^{n}$, is planned for the future.

\section{STATEMENT OF THE PROBLEM}

In this paper, if our system exhibits local smoothing properties, the result is that the solution belongs to the class Gevrey- $\delta$, where this Gevrey class is defined with respect to the time variable $t$. We formulation this more precisely in the definition below.

Definition $A$ function $f(x, t)$ belongs to the space $\gamma^{\delta}$ (Gevrey- $\delta$ class) uniformly for $(x, t)$ in a compact set $K$ if, for every $(x, t) \in K$ and for every $\theta>0$,

$\left|\frac{\partial^{j}}{\partial t^{j}} f(x, t)\right| \leq C_{K, \theta} \theta^{j}(j !)^{\delta}$

Our study of Schrödinger equations with nonconstant coefficients in the principal part of the differential operator begins with the following system.

$$
\begin{aligned}
i \frac{\partial u}{\partial t}+\mathcal{A} u & =0, \quad x \in \mathbf{R}^{n}, \quad t>0, \\
u(x, 0) & =\phi(x),
\end{aligned}
$$

where

$\mathcal{A} \equiv \sum_{i, j=1}^{n} \frac{\partial}{\partial x_{i}} a_{i j}(x) \frac{\partial}{\partial x_{j}}+c(x)$.

Note that, by definition, any potential is included in the operator $\mathcal{A}$. 


\subsection{Main results}

In Horn and Littman (1995), the authors prove the following regularity theorem.

Theorem 1 Assume the coefficients of $\mathcal{A}$ are real and satisfy the following conditions:
i) $a_{i j}(x)=a_{j i}(x)$
ii) $\sum_{i, j=1}^{n} a_{i j}(x) \xi_{i} \xi_{j} \geq \alpha|\xi|^{2} \quad$ for some $\alpha>0$,
iii) $a_{i j}(x)-\delta_{i j}, c(x) \in C_{0}^{\infty}\left(\mathbf{R}^{n}\right)$,

where $\delta_{i j}$ is the Kronecker delta. Without loss of generality, we assume that the support of these functions is contained in the ball $B_{a} \equiv\{x:|x|<a\}$.

Assume $\phi(x) \in L^{2}\left(\mathbf{R}^{n}\right)$ and has compact support. Then the solution $u(x, t)$ to the initial value problem (1) is of class Gevrey-2 with respect to $t$ uniformly in compact sets of $\mathbf{R}^{n} \times\{t>0\}$.

Our goal is to reduce the smoothness assumptions on the coefficients of $\mathcal{A}$. In Littman and Taylor (1992), the potential, $c(x)$, needed only to be bounded to obtain regularity results for the solution when $\mathcal{A} \equiv \Delta$. However, we also wish to reduce assumption iii) of Theorem 1 from $a_{i j}(x)-\delta_{i j} \in C_{0}^{\infty}\left(\mathbf{R}^{n}\right)$ to $a_{i j}(x)-\delta_{i j} \in C_{0}\left(\mathbf{R}^{n}\right)$, thus proving the following theorem.

Theorem 2 Assume the coefficients of $\mathcal{A}$ are real-valued, satisfy conditions i.) and ii.) of Theorem 1, and

iii) ${ }^{\prime} a_{i j}(x)-\delta_{i j}, c(x) \in C_{0}\left(\mathbf{R}^{n}\right)$ for all $i, j$.

Assume $\phi(x) \in L^{2}\left(\mathbf{R}^{n}\right)$ and has compact support. Then the solution $u(x, t)$ to the initial value problem (1) is of class Gevrey-2 with respect to $t$ uniformly in compact sets of $\mathbf{R}^{n} \times\{t>0\}$.

The proof of the Gevrey regularity in time (as given in Littman and Taylor (1992) and Horn and Littman (1995)) consists of solving the Schrödinger equation by the Laplace transform and showing that the inversion integral can be transformed into a contour integral along the boundary of what is essentially a union of two parabolas in the left hand plan of the transformed time variable $\lambda$.

The justification of this contour change consists of studying the modified resolvent, $\tilde{R}\left(k^{2} ; \mathcal{A}\right)=\chi(x)\left(k^{2}+\mathcal{A}\right)^{-1} \chi(x)$, (where $\chi(x)$ is a cutoff function) and to show that $\tilde{R}\left(k^{2} ; \mathcal{A}\right)$ can be continued as a bounded analytic operator function into a set of the type

$\{k: \Im m k \geq-b, \Re e k>a\}$.

This has been done in Horn and Littman (1995) for $C^{\infty}$ coefficients. To study nonsmooth coefficients, a perturbation argument is used below. The function $\tilde{u}$ introduced below is essentially the continuation of the modified resolvent acting on $f$,

$\tilde{u}=\tilde{R}\left(k^{2} ; \mathcal{A}\right) f$. 
Thus, in this paper, we will focus on proving the modified resolvent exists and is bounded in this extended region. Once these bounds have been established, proof of the Gevrey regularity of the solution follows as in Horn and Littman (1995) and combines straightforward estimation with the semigroup theory of Pazy (1983).

\section{PROOF OF GEVREY REGULARITY OF THE SOLUTION}

In a sense, we will treat the case of $C_{0}\left(\mathbf{R}^{n}\right)$ coefficients as a perturbation of the $C_{0}^{\infty}\left(\mathbf{R}^{n}\right)$ case. However, this does not allow us to apply a standard perturbation argument because the perturbation occurs in the principal part of the differential operator. Similarly to the proof of Theorem 1, we begin with the Laplace transform of the system but after this, we quickly diverge from the proof found in Horn and Littman (1995).

\subsection{Step 1: Laplace transform}

Taking the Laplace transform of (1) with respect to $t$, we find

$$
\begin{aligned}
i \lambda \tilde{u}-\mathcal{A} \tilde{u} & =i \phi(x) \\
\text { or } \mathcal{A} \tilde{u}+k^{2} \tilde{u} & =i \phi(x) \equiv f(x)
\end{aligned}
$$

where $\lambda=-i k^{2}$. Define $\mathcal{L} \equiv \mathcal{A}+k^{2}, \mathcal{L}_{0} \equiv \Delta+k^{2}$. As in Theorem 1 , without loss of generality, we assume $a_{i j}(x) \equiv 1$ outside a ball of radius $a, \mathcal{B}_{a} \equiv\{x:|x|<a\}$, and that the supports of both $c(x)$ and $\phi(x)$ are contained in $\mathcal{B}_{a}$.

\subsection{Step 2: Representation of solutions}

To facilitate the proof, we impose an "artificial" boundary condition, $\left.\mathcal{B} u\right|_{\partial \mathcal{B}_{a+3 \varepsilon}}=0$, on the system 5 . This boundary condition could be, for example, either a Dirichlet or Neumann boundary condition. In order to localize the solution inside and outside of $\mathcal{B}_{a}$, we define the following two cutoff functions, $\psi(x), \zeta(x) \in C^{\infty}\left(\mathbf{R}^{n}\right), 0 \leq \psi(x), \zeta(x) \leq 1 \forall x \in \mathbf{R}^{n}$, as follows.

$$
\psi(x)=\left\{\begin{array}{ll}
0 & |x| \leq a \\
1 & |x| \geq a+\epsilon
\end{array} \quad \zeta(x)= \begin{cases}1 & |x| \leq a+2 \epsilon \\
0 & |x| \geq a+3 \epsilon\end{cases}\right.
$$

and let $w=\psi \tilde{u}$ (outer solution) and $v=\zeta \tilde{u}$ (inner solution). Then $\tilde{u}$ can be written in terms of $w$ and $v$.

$$
\begin{aligned}
\tilde{u} & =(1-\psi) \tilde{u}+\psi \tilde{u}=(1-\psi) \tilde{u}+w \\
& =(1-\psi)(1-\zeta) \tilde{u}+(1-\psi) \zeta \tilde{u}+w \\
& =(1-\psi) v+w
\end{aligned}
$$

since $(1-\psi)(1-\zeta) \equiv 0$ for all $x \in \mathbf{R}^{n}$. 
Applying $\mathcal{L}$ to $w$ and $v$, we find

$$
\begin{aligned}
& \mathcal{L} w=\mathcal{L}_{0} w=\psi \mathcal{L} \tilde{u}+[\mathcal{L}, \psi] \tilde{u}=[\mathcal{L}, \psi] v \\
& \mathcal{L} v=\zeta \mathcal{L} \tilde{u}+[\mathcal{L}, \zeta] \tilde{u}=f+[\mathcal{L}, \zeta] w
\end{aligned}
$$

where $[\cdot, \cdot]$ denotes the commutator of the two operators and where $\psi$ and $\zeta$ also represent multiplication operators. Solving for $w$, we find

$w=\mathcal{L}^{-1}[\mathcal{L}, \psi] v=\mathcal{L}_{\text {ext }}^{-1}\left[\mathcal{L}_{\text {int }}, \psi\right] v$

$\Longrightarrow v=\mathcal{L}_{\text {int }}^{-1}\left\{f+\left[\mathcal{L}_{\text {ext }}, \zeta\right] \mathcal{L}_{\text {ext }}^{-1}\left[\mathcal{L}_{\text {int }}, \psi\right] v\right\}$,

where

$$
\mathcal{L}_{\text {int }} \equiv \mathcal{L}, \quad \mathcal{D}\left(\mathcal{L}_{\text {int }}\right) \equiv H_{0}^{1}\left(\mathcal{B}_{a+3 \epsilon}\right), \quad \mathcal{L}_{\text {ext }} \equiv \mathcal{L}_{0}, \quad \mathcal{D}\left(\mathcal{L}_{\text {ext }}\right) \equiv H^{1}\left(\mathbf{R}^{n}\right)
$$

We note for future reference that, with the above definition, $\mathcal{L}_{\text {int }}$ is constant outside of $\mathcal{B}_{a}$.

\subsection{Approximation of continuous coefficient problem}

At this point, in order to differentiate between the problem with $C^{\infty}$ coefficients and continuous coefficients, we define the following notation.

- $\mathcal{A}_{\infty}$ and $\mathcal{A}_{c}$ are defined as in (2) with $C^{\infty}$ and continuous coefficients, respectively.

- $\mathcal{L}_{\infty} \equiv \mathcal{A}_{\infty}+k^{2}, \mathcal{L}_{c} \equiv \mathcal{A}_{c}+k^{2}$.

We choose the coefficients of $\mathcal{A}_{\infty}$ such that given $\epsilon>0, \max _{x}\left|a_{\infty, i j}(x)-a_{c, i j}(x)\right|<\epsilon$. Thus, $\mathcal{A}_{\infty}$ can be considered to be a perturbation of the operator $\mathcal{A}_{c}$.

\subsection{Estimation of the inner solution}

Define

$M_{\infty} \equiv\left[\mathcal{L}_{0}, \zeta\right] \mathcal{L}_{0}^{-1}\left[\mathcal{L}_{\infty, \text { int }}, \psi\right] \quad M_{c} \equiv\left[\mathcal{L}_{0}, \zeta\right] \mathcal{L}_{0}^{-1}\left[\mathcal{L}_{c, \text { int }}, \psi\right]$

Then

$$
\begin{aligned}
& v_{\infty} \equiv \mathcal{L}_{\infty, \text { int }}^{-1}\left\{f+M_{\infty} v_{\infty}\right\} \quad v_{c} \equiv \mathcal{L}_{c, \text { int }}^{-1}\left\{f+M_{c} v_{c}\right\} \\
& \Longrightarrow \quad \begin{aligned}
v_{c}-v_{\infty} & =\left(\mathcal{L}_{c, \text { int }}^{-1}-\mathcal{L}_{\infty, \text { int }}^{-1}\right) f+\mathcal{L}_{c, \text { int }}^{-1} M_{c} v_{c}+\mathcal{L}_{\infty, \text { int }}^{-1} M_{\infty} v_{\infty} \\
& =\delta \mathcal{L}_{\text {int }}^{-1} f+\delta \mathcal{L}_{\text {int }}^{-1} M_{c} v_{c}+\mathcal{L}_{\infty, \text { int }}^{-1} \delta M v_{c}+\mathcal{L}_{\infty, \text { int }}^{-1} M_{\infty} \delta v
\end{aligned} \\
& \Longrightarrow \quad\left(I-\mathcal{L}_{\infty, \text { int }}^{-1} M_{\infty}\right) \delta v=\delta \mathcal{L}_{\text {int }}^{-1} f+\delta \mathcal{L}_{\text {int }}^{-1} M_{c} v_{c}+\mathcal{L}_{\infty, \text { int }}^{-1} \delta M v_{c},
\end{aligned}
$$

where

$$
\delta v \equiv v_{c}-v_{\infty}, \quad \delta M \equiv M_{c}-M_{\infty}, \quad \delta \mathcal{L}_{\text {int }}^{-1} \equiv \mathcal{L}_{c, \text { int }}^{-1}-\mathcal{L}_{\infty, \text { int }}^{-1} .
$$


By definition, we have chosen $\mathcal{L}_{\infty, \text { int }}$ such that

$\mathcal{L}_{\infty, \text { int }} \equiv \mathcal{L}_{c, \text { int }}+\mathcal{P}$

where $\mathcal{P}$ is a small perturbation of the differential operator. Thus,

$$
\begin{aligned}
\mathcal{L}_{\infty, \text { int }}^{-1}-\mathcal{L}_{c, \text { int }}^{-1} & =\mathcal{L}_{\infty, \text { int }}^{-1}-\left(\mathcal{L}_{\infty, \text { int }}+\mathcal{P}\right)^{-1}=\mathcal{L}_{\infty, \text { int }}^{-1}-\left(\mathcal{L}_{\infty, \text { int }}+\mathcal{P} \mathcal{L}_{\infty, \text { int }}^{-1} \mathcal{L}_{\infty, \text { int }}\right)^{-1} \\
& =\mathcal{L}_{\infty, \text { int }}^{-1}-\left[\left(I+\mathcal{P} \mathcal{L}_{\infty, \text { int }}^{-1} \mathcal{L}_{\infty, \text { int }}\right]^{-1}\right. \\
& =\mathcal{L}_{\infty, \text { int }}^{-1}-\mathcal{L}_{\infty, \text { int }}^{-1}\left(I+\mathcal{P} \mathcal{L}_{\infty, \text { int }}^{-1}\right)^{-1} \\
& =\mathcal{L}_{\infty, \text { int }}^{-1}\left\{I-\left(I+\mathcal{P} \mathcal{L}_{\infty, \text { int }}^{-1}\right)^{-1}\right\}
\end{aligned}
$$

Since $\|\mathcal{P}\|_{H^{1} \rightarrow H^{-1}}<\epsilon, I+\mathcal{P} \mathcal{L}_{\infty, \text { int }}^{-1} \sim I$, therefore,

$\left\|\mathcal{L}_{c, \text { int }}^{-1}-\mathcal{L}_{\infty, \text { int }}^{-1}\right\|_{H^{-1} \rightarrow H^{1}}<\epsilon\left\|\mathcal{L}_{\infty, \text { int }}^{-1}\right\|_{H^{-1} \rightarrow H^{1}}$

Next, we need to bound $\delta M$. Rewriting $\delta M$, we find

$$
\begin{aligned}
\delta M \equiv M_{c}-M_{\infty} & =\left[\mathcal{L}_{0}, \zeta\right] \mathcal{L}_{0}^{-1}\left[\mathcal{L}_{c, \text { int }}, \psi\right]-\left[\mathcal{L}_{0}, \zeta\right] \mathcal{L}_{0}^{-1}\left[\mathcal{L}_{\infty, \text { int }}, \psi\right] \\
& =\left[\mathcal{L}_{0}, \zeta\right] \mathcal{L}_{0}^{-1}\left[\mathcal{L}_{c, \text { int }}-\mathcal{L}_{\infty, \text { int }}, \psi\right]
\end{aligned}
$$

Note that in the above equation,

$$
\begin{aligned}
& \operatorname{supp}\left[\mathcal{L}_{c, \text { int }}, \zeta\right] \subset \mathcal{B}_{a+3 \epsilon} \backslash \mathcal{B}_{a+2 \epsilon} \\
& \operatorname{supp}\left[\mathcal{L}_{c, \text { int }}, \psi\right] \subset \mathcal{B}_{a+\epsilon} \backslash \mathcal{B}_{a} \\
& \operatorname{supp}\left[\mathcal{L}_{c, \text { int }}-\mathcal{L}_{\infty, \text { int }}, \psi\right] \subset \mathcal{B}_{a+\epsilon} \backslash \mathcal{B}_{a}
\end{aligned}
$$

and we know, by definition, that $\mathcal{L}_{c} \equiv \mathcal{L}_{\infty}$ in $\mathcal{B}_{a+3 \epsilon} \backslash \mathcal{B}_{a}$. Therefore, $\delta M \equiv 0$.

Combining the results for $\delta \mathcal{L}_{\text {int }}^{-1}$ and $\delta M$, we find that for some constant $C_{v}>0$,

$\left\|v_{c}-v_{\infty}\right\|_{L^{2}}<\epsilon C_{v}\left\|\mathcal{L}_{\infty, \text { int }}^{-1}\right\|_{H^{-1} \rightarrow H^{1}}$

We note that the constant $C_{v}$ (and $C_{w}, C_{u}$ that follow in the next section) is notational only and does not imply any dependence on $v$.

\subsection{Estimates for $\tilde{u}_{c}-\tilde{u}_{\infty}$}

Using equation (8), we find

$$
\begin{aligned}
& \begin{aligned}
w_{c}-w_{\infty} & =\mathcal{L}_{0}^{-1}\left[\mathcal{L}_{c, \text { int }}, \psi\right] v_{c}-\mathcal{L}_{0}^{-1}\left[\mathcal{L}_{\infty, \text { int }}, \psi\right] v_{\infty} \\
& =\mathcal{L}_{0}^{-1}\left\{\left[\mathcal{L}_{c, \text { int }}-\mathcal{L}_{\infty, \text { int }}, \psi\right] v_{c}-\left[\mathcal{L}_{\infty, \text { int }}, \psi\right]\left(v_{c}-v_{\infty}\right)\right\}
\end{aligned} \\
& \Longrightarrow\left\|w_{c}-w_{\infty}\right\|_{L^{2}}<\epsilon C_{w}\left\|\mathcal{L}_{\infty, \text { int }}^{-1}\right\|_{H^{-1} \rightarrow H^{1}} \quad \text { for some constant, } C_{w}>0 .
\end{aligned}
$$

Therefore, from (7), there exists a constant, $C_{u}>0$, such that

$$
\left\|\tilde{u}_{c}-\tilde{u}_{\infty}\right\|_{L^{2}}<\epsilon C_{u}\left\|\mathcal{L}_{\infty, \text { int }}^{-1}\right\|_{H^{-1} \rightarrow H^{1}}
$$




\subsection{Step 6: Phragmén-Lindelöf theorem}

From the proof of Theorem 1 in Horn and Littman (1995), if the coefficients of problem (1) are $C^{\infty}$, we know that the modified resolvent corresponding to this problem satisfies

$$
\left\|\tilde{R}\left(k^{2} ; \mathcal{A}_{\infty}\right)\right\|_{H^{-1} \rightarrow H^{1}}<C \quad \forall k \in \mathcal{T}_{a, b} \equiv\{k: \Im m k \geq-b,|k| \geq a\} .
$$

Hence, in particular, the inequality in (21) implies

$$
\left\|\tilde{R}\left(k^{2} ; \mathcal{A}_{c}\right)\right\|_{H^{-1} \rightarrow H^{1}}<C \quad \forall k \in\{k:|\Im m k|=b, \Re e k=a\},
$$

i.e., the modified resolvent associated with $\mathcal{A}_{c}$ is uniformly bounded with respect to $k$ on the boundary of the strip

$$
\{k:|\Im m k| \leq b, \Re e k>a\} .
$$

In deriving the boundedness of $v$ as a function of $k$, the boundedness of $\mathcal{L}_{c}^{-1}$ as a function of $k$ is needed. Unfortunately, there is a sequence of poles (corresponding to the eigenvalues of the Dirichlet problem for $\mathcal{A}_{c}$ ) which prevents the direct estimation of the modified resolvent in the strip. From the results of Birman and Solomjak (1972) on the distribution of eigenvalues, it follows that we can find a sequence of vertical line segments in the strip on which bounds for the modified resolvent will increase no faster than a power of $\Re e k$. Then an application of the Phragmén-Lindelöf Theorem (see Titchmarsh (1939), section 5.65) gives the boundedness of $R\left(k^{2} ; \mathcal{A}\right)$ in the entire strip.

\section{REFERENCES}

Bardos, C., Lebeau, G. and Rauch, J. (1989) Contrôle et stabilisation dans les problèmes hyperboliques. In Contrôlabilité exacte, perturbations et stabilization de systèmes distribués, volume 2, Paris. Masson. Appendix 2.

Birman, M. S. and Solomjak, M. Z. (1972) Spectral asymptotics of nonsmooth elliptic operators. Soviet Math. Dokl., 13(2):906-910.

Horn, M. A. and Littman, W. (1995) Boundary control of a Schrödinger equation with nonconstant principal part. In Control of Partial Differential Equations and Applications, Lecture Notes in Pure and Applied Mathematics, Marcel Dekker, New York.

Lasiecka, I. and Triggiani, R. (1992) Optimal regularity, exact controllability and uniform stabilization of Schrödinger equations with Dirichlet control. Differential and Integral Equations, 5:521-535.

Lebeau, G. (1992) Contrôle de l'equation de Schrödinger. Journal Math. Pures Appl., $71: 267-291$.

Littman, W. and Taylor, S. (1992) Smoothing evolution equations and boundary control theory. Journal D'Analyse Mathématique, 59:117-131.

Pazy, A. (1983) Semigroups of Linear Operators and Applications to Partial Differential Equations. Springer-Verlag, New York.

Titchmarsh, E. C. (1939) The Theory of Functions. Oxford University Press, London. 